\title{
The Reform of Value Logic of Minority Areas' Public Hospitals at the County Level: Taking Ethnic Minority in Gansu Province as an Example
}

\author{
Yilong Wang ${ }^{1, a^{*}}$ and Haiying $\mathrm{Ma}^{2, \mathrm{~b}}$ \\ 1, School of Management, Northwest University for Nationalities, Lanzhou (730124), P.R.China \\ ${ }^{2}$ School of Economics, Northwest University for Nationalities, Lanzhou (730124), P.R.China \\ awyl19881129@163.com, 'lxmahaiying8888@163.com
}

\begin{abstract}
Keywords: Race region; Public hospital reform; Content of logic; Practical dilemma; Value logic
\end{abstract}
\begin{abstract}
Reform of public hospitals at the county level in national regions is different from the actual situation of other administrative regions, taking minority areas in Gansu province as an example, combing the current content logic on the basis of the reform of public hospitals at the county level in China, studying the current practical dilemma of the current reform of public hospitals at the county level in China, and putting forward the public interests, fairness, justice and public choice is the value logic that public hospital at the country level in Gansu province and national minority areas should follow.
\end{abstract}

\section{Introduction}

County-level public hospitals are the link between the rural tertiary health care service network and the urban and rural medical and health service system. Promoting the comprehensive reform of the county-level public hospitals is the key to deepening the reform of the medical and health system and effectively alleviating the problem of difficult and expensive doctor of the masses. Since the start of the comprehensive reform of county public hospitals in 2012, China's pilot counties (cities) have been actively exploring and the reform has made remarkable progress. The reform of county-level public hospitals, which is related to the life safety of the population of more than $50 \%$ of our country, is directly related to the fairness and justice of our public hospital reform performance. Ethnic areas as an important administrative region of our country, the significance of the public hospital's reform may be given greater prominence and decides whether China's 56 nationalities can benefit from it or not. China's minority areas is very large and composition is complex, every nationalities' life areas has obvious differences, different location of minority areas' public hospital at the country level may face the different problems and needs. So it's not suitable to standardized carrying out the institutional supply and it's suitable to designing an appropriate way that take the different nationalities' different cultures, economic and social development, geographical location and religious beliefs into account. This research takes the Gansu province as an example, which minority areas is large and composition is complex, to studying the value logic of China's minority areas' public hospital reform at the country level.

Gansu Province is a multi-ethnic inhabited place. The province's existing 55 ethnic minorities, the minority population of 2.4105 million people, accounted for the total population of $9.43 \%$, the country area of 180,000 square kilometers, which accounting for $39.8 \%$ of the total area of the province. Gansu province's ethnic composition is complicated and minorities' religion beliefs are huge different. Reform of public hospital at the country level significantly influences the life safety of ethnic minority areas' people and even the stabilization of regional situation.

Reform of Gansu province ethnic minority areas' public hospital at the country and reform of China ethnic minority areas' public hospital at the country level is significantly different from non-multi-nationality areas' public hospital at the country, so if we follow the logical content of the other provinces, it will lead to the imperfect outcome performance of the reform. It is wise to adopting the different methods for different ethnic minority areas about public hospital reform at the country. But the value logic should be clear and reasonable, Going against the real condition of the ethnic minority areas' 
reform value logic will be probably endangering the social stability and harmony of ethnic minority areas.

\section{The Logical Content of the Current Public Hospital Reform at the Country Level}

Since June 2012, General Office of the State Council has noticed The opinions of public hospitals' reform at the country level pilot notice, the country government gradually carried out various forms of reform pilot on the basis of their own actual situation. China's public hospitals' reform at the country level mainly focused on the reform of the public hospitals' administration patterns, such as Zichang Country of Shaanxi Province. Zichang Country of Shaanxi Province's public hospital reform logic at the country level focused on the personnel system, which mainly concerned on the reform of personnel distribution system innovation and implemented full employment. The direct value of the reform is medical staff income increasing from 29000 Yuan to 80000 Yuan and highest can reach 200000 Yuan. Sanming City of Fujian Province's public hospital reform logic at the country level focused on Distribution mechanism, compensation mechanism, appraisal mechanism, drug purchasing, hospital management, fund management and so on. The first step of Kunming city of Yunnan Province's public hospital reform logic at the country level is canceling the drug addition, the second step is ensuring the regular investment from government to public hospital at the country level, the third step is increasing the talent construction and introduction, the forth step is reforming the public hospital management system. Zhuzhou city of Hunan Province's public hospital reform logic at the country level positions in adjusting the medical service prices, Start the medical insurance payment reform and implementation of government investment responsibility and so on.

Domestic scholars' major research direction on public hospital reform logic at the country level reflects in experiences and achievements of public hospital reform at the country level. Haihong Zhang has researched achievements of Zhejiang Province's public hospital reform and put forward the main mode that Zhejiang Province should establish the basic drug system, break medical benefit chain, adjust the structure of medical service charge, promote the value of medical staff labor, Strengthen the financial safeguard and reinforce the government's responsibility, strengthen the hospital internal management, strictly control all expenses and so on. Reform value of Zhejiang Province reflected in public hospitals breaking the medical benefit chain and controlling the excessively rapid growth of medical expenses, adjusting the medical service peices and highlighting the technical labor value of medical personnel, making the medical staff reasonable income increased and inspiring the enthusiasm of medical staff to improve the service and quality, decreasing old patients and chronic patients' drug expenses, improving patients satisfaction. Dupeng has researched Zichang country in Shaanxi Province and put forward the public hospitals reform's main logic of Zichang country in Shaanxi Province is reforming the financing channels, internal mechanism, external hospital management. Reform has made public hospital of Zichang country gradually return to the nature of public welfare, the country residents' enthusiasm improved, drug proportion observably declined. He Rong has researched Henan Province's public hospitals reform at the country level and put forward it's main reform concerns that focus on public hospital drugs zero sales and reform of the medical fee pricing mechanism, implement the cost control function of health insurance and DRGs pay pattern, promote the reform of public hospital corporate governance structure, implement The first treatment and settlement medical service model for hospitalized patients, establish the two-way referral system and country, township and village level collaboration mechanism and so on.

After observing the reform content logic of public hospital at the country level, we find it's main idea focusing on the public administration pattern reform of public hospital at the country level and it's logic starting point fundamentally following the principle that reform process should reflect people's value aim. Therefore, from the perspective of the feedback of the performance evaluation, Ministry of Health highly affirms the public hospitals' reform results at the country. From the perspective of the perspective of the performance results, it basically contains rapid medical costs growth, breaks the "drug-maintaining-medicine" mechanism, improves the tense doctor-patient relationship, solves the problem that it is difficulty and expensive for commom people to see a doctor. 


\section{The Realistic Predicament of Minority Areas' Public Hospital Reform at the Country Level}

\section{Identity Disorders of the Public Hospitals' Reform under the Background of Multi-ethnic} Cultures. Ethnic minorities lived in Gansu Province for generations is Hui, Zang, Dongxiang, Baoan, Yugu, Mongol, Salar, Kazak, Tu, Manchu. Dongxiang, Baoan, Yugu nationlaity is peculiar living in Gansu Province. From the view of ethnic composition, ethnic composition of Gansu Province is complex. From the view of group culture, it is directly reflected in the conflict of multicultural values. It is classified as religion for 10 ethnic minorities in Gansu Province. Hui, Dongxiang, Baoan, Salar ,Kazak main faith is Islam. Zang, Yugu, Tu main faith is Tibetan Buddhism. Tibetan Buddhism is part of the Mongolian belief, and part of Mongol nationality main faith is Shamanism. Manchu nationality mainly migrates from China's northeast and main faith is Shamanism.

In the process of the public hospital's reform at the country level, it will bring about culture obstruct if act in accordance with the national and provincial government document standardization. Gansu Province people's government has issued 《Gansu Province public hospital at the country level reform plan notification》(Gansu Province government do hair[2015]no.144).In this government notice part of optimizing the country medical resources, Gansu Province government has put forward the suggestion that provincial health department should select appropriate technology to apply to public hospital at the country level. But in the process of practically generalizing, it may be hampered by a multi-cultural value conflict in national minority areas. Such as Zang nationality of Gansu Province mainly distributed in Tibetan Autonomous Prefecture of Gannan and Tianzhu Tibetan autonomous country, it may cause reject from local Tibetan if enforce traditional Chinese medicine appropriate technology during the public hospital reform at the country level and main reason of this problem is that Tibetan has its own unique Tibetan medicine system. Tibetan medicine system is combined with the conditions of high altitude, Tibetan diet culture and Tibetan body system, so it form the profound cultural foundation and value identity in Tibetan areas. If government enforces traditional Chinese medicine, it will cause resentment of Tibetan and not adapt to the needs of local medical and health, finally result in the loss of performance of country public hospital reform.

Country Public Hospital Reform under the Background of Lack Of Social Security System. The ethnic areas in Gansu province including 2 autonomous prefectures(Gannan Tibetan Autonomous Prefecture, Linxia Hui Autonomous Prefecture), 7 autonomous counties (Zhangjiachuan Hui Autonomous County, Tianzhu Tibetan Autonomous County, Subei Mongolian Autonomous County, Sunan Yugur Autonomous County, Aksay Kazak Autonomous County, Dongxiang Autonomous County and Jishishan Salar Autonomous County of Cheyenne Dongxiang nationality).Total population of this area is 3 million 331 thousand people, accounting for the province's $13.02 \%$ total popularion. Minority population of this area is 1 million 993 thousand people and land area is 180 thousand square kilometers, accounting for the province's 39.8\% total area. In addition, Gansu Province has 35 ethnic townships, which includes 16 Hui nationality townships, 8 Dongxiang nationality townships, 7 Tibetan nationality townships, 1 Yugur township,2 Mongol nationality townships, 1 Tu nationality township, total population is 311 thousand people including 206 thousand minority people.

From the view of distribution, Hui nationality mainly lives in Linxia Hui Autonomous Prefecture and Zhangjiachuan Hui Autonomous Country, scatters in Lanzhou, Pingliang, Dingxi and other cities. Zang nationlaity mainly lives in Gannan Tibetan Autonomous Prefecture and eastern and middle parts of the Qilian Mountain in the Hexi Corridor. Dongxiang, Baoan, Sala nationality mainly distributes in Linxia Hui Autonomous Prefecture.Yugu, Mongol, Kazak mainly distributes in the western areas of Qilian Mountain in the Hexi Corridor.

In recent years, social security of ethnic minority areas in Gansu province has made some achievements, but compared with other regions in Gansu province, social security function of ethnic minority areas is relatively lacking. 12 ethnic countries in Gansu province have be listed as national and provincial poverty counties and poor population in ethnic areas accounts for more than half. Gansu ethnic areas NCMS participation rate has continued to increase in recent years, social assistance system has continuously improved. However, it has been affected by the economic, social, historical and other aspects for a long 
time, negative externalities of urbanization and industrialization, Gansu ethnic areas' stability and regulation degree of social security is relatively low.

It is shown in the second quarter of 2015 Gansu province medical and health service statistics report that Aksay Kazak Autonomous County ranks last in country medical and health institution rank, patients number in this period is 9700.Subei Mongolian Autonomous Country ranks second in the second quarter of 2015.Diebu Country, Zhouqu Country, Luqu Country, Maqu Country, Xiahe Country in Gannan Tibetan Autonomous Prefecture ranks later in ranking. Sunan Yugur Autonomous ranks last eighth in the second quarter of 2015 and patients number is 28900.

Statistical date can also be regarded as the direct result of the policy output. The direct result of the instability of the social security is the lack of the function of public hospital at the country level. Farmers and herdsmen are the main part of Gansu ethnic areas, the percentage of reimbursement for medical reimbursement for this group is not high and many people are even not participating in medical insurance. Therefore, when you encounter the risk of disease, national medical policy awareness is not high, the medical expense burden of fear of common cause the county public hospitals to visit the volume is not high, the discharge rate is not high, resulting in a domino effect, and jointly with the county-level public hospitals are weak diagnosis and treatment capacity, medical technology backward, health professionals lack.

Financial Burden of Country Public Hospital Reform under the Background of Underdeveloped Economic Development. Poor people in Gansu province is 8 million 375 thousand and 200 people, accounting for $40.12 \%$ of the province's rural population. Population of ethnic areas is 1 million 427 thousand and 700, accounting for the province's poor population of about $17 \%$ and total population of rural areas of $51 \%$ country population. Gannan Tibetan Autonomous Prefecture poor population is 283 thousand and 900 people, accounting for about $51.29 \%$ of the total rural population. Linxia Hui Autonomous Prefecture poor population is 900 thousand and 200 people, accounting for about 52.04\% of the total rural population. Tianzhu Tibetan Autonomous Country poor population is 87 thousand and 600 ,accounting for $50.85 \%$ of the total population of the total rural population. Zhangjiachuan Hui Autonomous Country poor population is 153 thousand and 300 people, accounting for the country's 49.34\% total rural population. 16 Autonomous Countries in Gansu Province per capital net income of farmers is lower than the national average, 5 Autonomous Country's population is more than 100 thousand people.18 Autonomous Countries are included in the national poverty areas ,accounting for $85 \%$ of the total national poor areas.

Reform of public hospital's main goal is to get rid of the mechanism of covering hospital expenses with medicine revenue, change the financial compensation mechanism, and extend to the public hospital management system, operating mechanism, medical insurance payment system, personnel management system, Income distribution system and grading system reform. The status of relative poverty in ethnic areas has directly caused the tension of the country finance, and it's direct consequence is the weakness of the local people's ability to see a doctor. Therefore, the public hospital reform resistance of ethnic areas in Gansu Province and national ethnic areas is backward economic situation. Backward economic situation not only affects the administrative efficiency of the local government, but also directly affects the level of medical services in the country.

\section{Value logic of Ethnic Areas Public Hospital Reform in Gansu Province}

Public Interest. Public hospital reform is a reform of public administration, the only measure of performance standards should be in line with the public interest. George Fred Rick Sen defines the word "public" , "public" is a concept that covers a wide range of former government(Mathews, 1994).It is reflected in many aspects, such as neighborhoods, voluntary groups, churches, clubs and other human activities in many areas and decomposition of many public institutions. Government is an important department reflecting the public character, but not the only department(Bozeman, 1987).In order to understand public administration, it is necessary to rediscover the publicity and it must be understood from the view of "public administration". If we interpret "public interest" from the utilitarian perspective, 
it should be understood that "the greatest happiness of most people", but also can be understood a process that not affect and harm other people's benefit when one does one thing. Any reform take "public interest" as value logic can be seen as a system with the nature of public goods supply, Gansu Province's public hospital reform is no exception. The focus of reform, wether it is from the management system, operation mechanism, the medicare payment system, personnel management system, income distribution system, grading treatment system, should fully reflect the interests of ethnic areas in Gansu Province. The purpose of reform is to benefit majority people.

Fairness and Justice. Fairness and justice is the ultimate value proposition since the human society has began. Gansu Province ethnic areas public hospital reform is without exception and value logic is to take the interests of fairness and justice into account. Rawls proposed the concept Veil of ignorance in Justice Theory. Veil of ignorance is an original state that members in this organization do not know their birth, social status, gender and natural talent. In this state, social status is fair for individuals. Ethnic areas contradiction is complex and directly consequence may be lead to mass disturbances and terrorist incident. Compared with China eastern region, economic development level and social civilization of ethnic areas is relatively backward. Therefore, it may lead to psychological and emotional maladjustment, and the failure of ethnic areas public hospital reform at the country level. Fairness and justice is the inevitable value logic of Gansu Province ethnic areas public hospital reform at the country level and the whole nation's public hospital reform at the country level. How to design a fair and justice reform path, it can refer to Rawls's two principles of justice, that is the principle of fair and equal opportunity and difference principle.

Public Choice. The theory of public choice rises in 1950s, core concept is to study collective decision-making with economic methods. The theory of public choice advocates that collective decision-making should fully consider the value preference and the needs of the majority people.

As a kind of supply system that has the property of public goods, the value logic of Gansu Province ethnic areas public hospital reform should be non-excludability and noncompetitive, which means Gansu Province public hospital reform should fully respect the choice and the value preference from different nationalities.

\section{Conclusion}

Another obvious characteristics of Gansu Province public hospital reform is the provincial administrative region of Gansu Province not a minority autonomous province. The provincial and national's ethnic composition is complex, distributed and multi-cultural. Therefore, considering the actual situation of ethnic countries public hospital reform in Gansu Province and common interests of all ethnic countries, value logic of ethnic areas public hospital reform is reasonable and effective. Gansu Province and the whole nation's public hospital reform at the country level should ensure fair and equal opportunities, increase the financial investment, cut off the mechanism of covering hospital expenses with medicine revenue. It is not a good way to copying the East Country public hospital reform mode and it is a good way to fully considering the actual situation of ethnic areas. Supply-side reform of ethnic areas should reflect the principle of fairness and justice.

\section{Acknowledgements}

This work was supported by the Fundamental Research Funds for the Central Universities of Northwest Minzu University (Grant No. 31920160021).

\section{References}

[1] National Health and Family planning System Reform Division. Notice on issuing the views of the comprehensive reform of the county-level public Hospitals, 12 (2014)77-81.

[2]Yu Fuchun and Mou Weiping, the Practice and enlightenment of the reform of county-level public hospitals in Shanxi Province, China health Policy Research, 5 (2012) 30-33. 
[3]Dou Lei, Chen Chun, Zhao Rong, China's public hospitals reform the policy analysis of national level, Chinese Hospital Management, 15 (2015)1-4.

[4]Chen Hang, Yang Rui and Wu Fan, the experience and discussion of the reform of public hospitals in Beijing, China Administration, 7 (2014)125-126.

[5]Chen Ji, A study of political stability in ethnic regions from the perspective of political system. Journal of Huazhong University of Science and Technology, 4 (2012)67-73

[6] David Iston, systematic analysis of political life. Beijing: People's publishing house. (2012)

[7] George Fred Rick Sen, the spirit of public administration, Renmin University of China press.(2003).

[8] Liu Junxiang, Yin Jinhua. Basic medical service reform in the perspective of justice theory[J].Philosophical analysis, 2(2013)108-115. 